\title{
Heterogeneidad de las células B de memoria IgM humanas
}

\author{
Juana Ángel \\ Instituto de Genética Humana, Facultad de Medicina, Pontificia Universidad Javeriana, Bogotá, Colombia
}

\begin{abstract}
Resumen
El origen, la función y el repertorio de las células B de memoria IgM humanas (caracterizadas por ser positivas para CD27 IgM e IgD) son controvertidos, y se ha propuesto que esta población es heterogénea. Aunque algunas veces contradictorias, las evidencias actuales apuntan a la existencia de por lo menos dos subpoblaciones de dichas células en sangre: por un lado, células B circulantes de la zona marginal del bazo, con algunas características similares a las células innatas y probablemente responsables de las respuestas de activación independiente de los linfocitos T, que protegen contra bacterias encapsuladas como Streptococcus sp, y, por otro lado, células B con indicios de haber pasado por centros germinales que se asemejan a las células B de la respuesta adaptativa, y que serían un reservorio de células B de larga vida a partir del cual se reconstituirían continuamente las células B de memoria conmutadas. Aunque se ha propuesto que la expresión diferencial de $\operatorname{IgM}$ e IgD en las células B de memoria IgM serviría para distinguir estas dos subpoblaciones de células B, se requieren más estudios fenotípicos y funcionales para sustentar esta clasificación.
\end{abstract}

Palabras clave: células B de memoria, IgM, respuesta B innata, respuesta B adaptativa.

Heterogeneity of human IgM memory B cells

\begin{abstract}
The origin, function and repertoire of human $\operatorname{IgM}$ memory B cells ( $\operatorname{IgM} \mathrm{mBc}$, characterized by the expression of $\mathrm{CD}_{27} 7^{+} \mathrm{IgM}^{+} \mathrm{IgD}^{+}$) are controversial, and it has been proposed that this population is heterogeneous. Although contradictory in some cases, available evidence suggests that at least two subpopulations of IgM $\mathrm{mBc}$ circulate in blood. On the one hand, circulating B cells from the marginal zone of the spleen with innate-like characteristics, probably responsible for protective responses against encapsulated bacteria like Streptococcus sp. On the other hand, B cells with markers that suggest they have undergone transit in germinal centers, typical of adaptive immunity, and that may represent a reservoir of long-lived $\mathrm{mBc}$ capable of reconstituting switched $\mathrm{mBc}$. It has been proposed that the differential expression of $\operatorname{IgM}$ and $\operatorname{IgD}$ on circulating $\operatorname{IgM~} \mathrm{mBc}$ may be useful to distinguish these subpopulations, but more phenotypic and functional studies are needed for this purpose.
\end{abstract}

Key words: Memory B cells, IgM, innate B cell response, adaptive B cell response.

\section{Introducción}

Ante una infección, las células $\mathrm{B}$ vírgenes (llamadas células B2 o linfocitos B2) se diferencian como células B de memoria $(\mathrm{cBm})$ o en células secretoras de anticuerpos específicas del patógeno con ayuda de los linfocitos $\mathrm{T}$ (respuesta inmune dependiente de $\mathrm{T}$ ). Las cBm que se generan durante esta respuesta adaptativa tienen varias características en común: larga vida, proliferación rápida $\mathrm{y}$ robusta en respuesta al antígeno, gran sensibilidad a bajas dosis de antígeno y diferenciación terminal rápida en células que secretan anticuerpos (Takemori, et al., 2014). Sin embargo, una fracción de las células B, catalogadas como células B innatas, expresan receptores $B$ que reconocen múltiples determinantes microbianos altamente conservados, se localizan en sitios anatómicos estratégicos que están en contacto con antígenos microbianos (Weller, et al., 2004), responden contra ellos en forma más rápida e independientemente de los linfocitos T (Cerutti, et al., 2013), y secretan anticuerpos naturales. Los anticuerpos naturales son IgM (circulantes y del bazo) o IgA (mucosas), y su estudio se inició en los ratones libres de gérmenes que tienen anticuerpos en ausencia de microorganismos, por lo que se propuso que su producción debía estar regulada de manera diferente a la de los anticuerpos específicos de microorganismos (Baumgarth, 2011). En ratones, tanto las células B1 (o linfocitos B1) como las células B de la zona marginal (cBZM) del bazo han sido implicadas en este tipo de respuestas innatas. En contraste, en los humanos las células B innatas son motivo de controversia, puesto que las células B1 como tal están mal caracterizadas

Correspondencia:

Juana Ángel, jangel@javeriana.edu.co

Recibido: 25 de octubre de 2015

Aceptado: 1 de febrero de 2016 
(Baumgarth, 2011; Reynaud \& Weill, 2012; Rothstein \& Quach, 2015), y las cBZM tienen algunas diferencias con las encontradas en los ratones. No obstante, Cerutti, et al. (2013) han propuesto que las cBZM humanas funcionan de forma similar a las innatas, o que sirven de puente entre la respuesta innata y la adquirida, en tanto que Weller, et al. (2004) han propuesto que constituyen la primera línea de defensa contra los microorganismos y que podrían secretar anticuerpos naturales.

Los conceptos en el campo de estudio de las cBm han evolucionado en la última década (Jackson, et al., 2008; Mroczek, et al., 2014). Comúnmente se ha aceptado que las $\mathrm{cBm}$ se originan con ayuda de los linfocitos $\mathrm{T}$ foliculares en los centros germinales, en los cuales se llevan a cabo cambios moleculares con el fin de optimizar la afinidad de las inmunoglobulinas por el antígeno. Estos cambios son el resultado de la activación de una enzima llamada desaminasa de citidina inducida por activación (activation-induced cytidine deaminase, AID), la cual participa en la generación de las hipermutaciones somáticas en las porciones variables del gen de las inmunoglobulinas ( $\mathrm{IgV}$ ) y en la conmutación de las inmunoglobulinas mismas. El concepto aceptado era que estos dos fenómenos ocurrían de forma secuencial (Pascual, et al., 1994) y solamente en el centro germinal, donde se encontraba la enzima activada. Actualmente se reconoce que existen $\mathrm{cBm}$ que probablemente no han pasado por el centro germinal y que no hacen conmutación de isotipo (Takemori, et al., 2014).
Teniendo en cuenta la expresión de CD27, IgD e IgM se han tipificado claramente cuatro subpoblaciones principales de cBm, (Lanzavecchia \& Sallusto, 2009; Mroczek, et al., 2014; Wirths \& Lanzavecchia, 2005) (Figura 1): 1) células $\mathrm{CD}^{+} \mathrm{IgM}^{-} \mathrm{IgD}^{-}$(cBm conmutadas, $\mathrm{IgG}^{+}, \mathrm{IgA}^{+}$o $\mathrm{IgE}^{+}$), que representan aproximadamente $20 \%$ de las células B circulantes; 2) células $\mathrm{CD}_{2} 7^{+} \operatorname{IgM}^{+} \operatorname{IgD}^{+}$(cBm $\operatorname{IgM}$ ), que constituyen aproximadamente $15 \%$ de las células $\mathrm{B}$ circulantes; 3) células $\mathrm{CD}^{2} 7^{+} \mathrm{IgM}^{+} \mathrm{IgD}^{-}$(cBm solo $\mathrm{IgM}$ ), que conforman aproximadamente $5 \%$ de las células B circulantes, y 4) células CD27- $\operatorname{IgM}^{-} \operatorname{IgD}^{-}$(cBm CD27), que suman entre 1 y $5 \%$ de las células B circulantes. En otros estudios se continuó el proceso de caracterización fina de estas células y actualmente se reconocen seis (Berkowska, et al., 2011b) o más poblaciones de cBm, porque se incluyen las poblaciones solo $\operatorname{IgD}^{+}\left(\mathrm{CD} 27^{+} \mathrm{IgM}^{-} \mathrm{IgD}^{+}\right)$ (Chen \& Cerutti, 2010) y se han encontrado diferencias para las células $\mathrm{CD} 27^{+} \mathrm{IgG}^{+}, \mathrm{CD}^{2} 7^{+} \mathrm{IgA}^{+}, \mathrm{CD}^{-} 7^{-} \mathrm{IgG}^{+} \mathrm{y}$ $\mathrm{CD}^{-} 7^{-} \operatorname{IgA}^{+}$, particularmente en cuanto al número de veces que han pasado por el centro germinal y al número de mutaciones en las porciones génicas que codifican para $\mathrm{IgV}$ (Berkowska, et al., 2011a).

De particular interés es la población de $\mathrm{cBm} \operatorname{IgM}$ circulante en humanos, pues los resultados de varios estudios sugieren que son una población heterogénea compuesta, probablemente, por al menos una población de células $\mathrm{B}$ con función adaptativa y con indicios de haber pasado
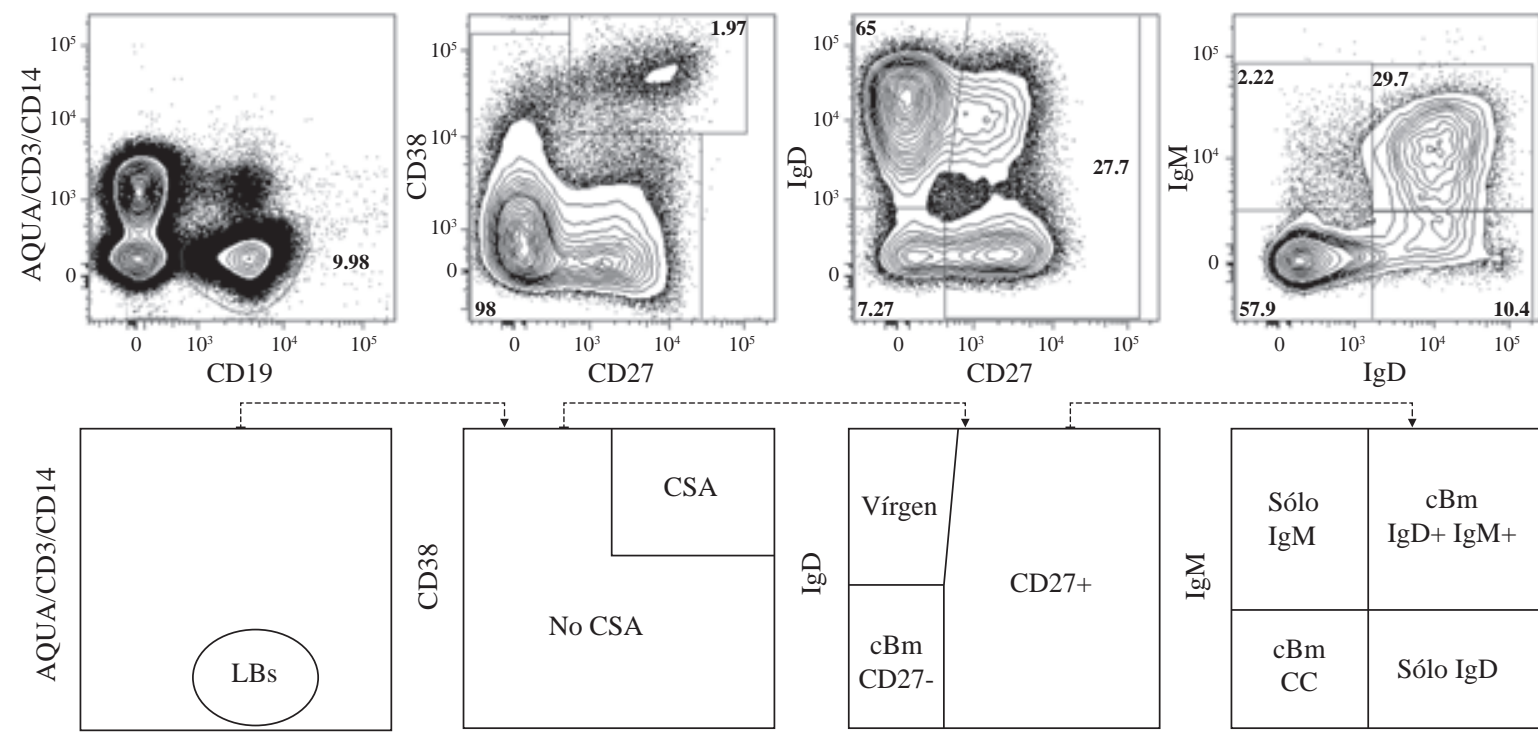

CD27

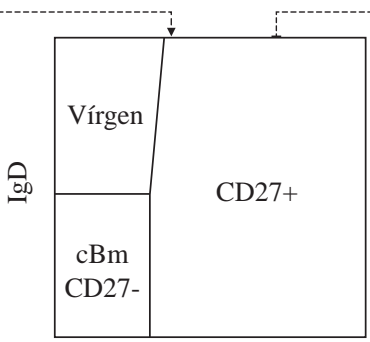

CD27

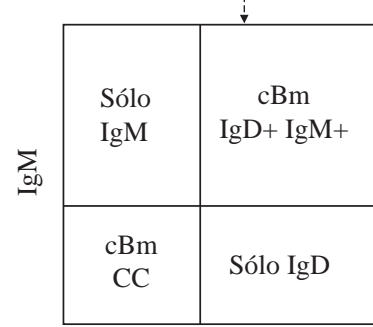

$\operatorname{Ig} \mathrm{D}$

Figura 1. Estrategia de identificación de las células B humanas circulantes en adultos mediante citometría de flujo. Células mononucleares de sangre periférica purificadas con gradiente de densidad teñidas con anticuerpos acoplados a diferentes fluorocromos y analizadas mediante citometría de flujo. Las células B se caracterizan por ser CD19+ $\mathrm{CD}^{-} \mathrm{CD} 14^{-}$; las células secretoras de anticuerpos $\left(\mathrm{CSA} \mathrm{CD}^{+8^{++}} \mathrm{CD} 27^{++}\right)$se excluyeron del análisis; con la expresión de IgD y CD27 se determinan las células B vírgenes (CD27- $\left.\operatorname{IgD}^{+}\right)$, las cBm CD27- $\left(\mathrm{CD}^{-7^{-}} \mathrm{IgD}^{-}\right)$ y las cBm CD27+. Al analizar la expresión de $\operatorname{IgM}$ e $\operatorname{IgD}$, únicamente en las $\mathrm{CD} 27+$, se identificaron las $\left.\mathrm{cBm} \operatorname{solo} \operatorname{IgM}\left(\mathrm{CD}_{2} 7^{+} \operatorname{IgM}{ }^{+} \operatorname{IgD}\right)^{-}\right)$, las cBm IgM $\left(\mathrm{CD} 27^{+} \operatorname{IgM}^{+} \operatorname{IgD}^{+}\right)$, las cBm solo $\operatorname{IgD}\left(\mathrm{CD} 27^{+} \operatorname{IgM}^{-} \operatorname{IgD}^{+}\right)$y las cBm conmutadas $\left(\mathrm{CC}, \mathrm{CD}^{2} 7^{+} \operatorname{IgM}^{-} \operatorname{IgD}^{-}\right)$. Los números en los recuadros representan los porcentajes de las poblaciones en cada ventana. 
por el centro germinal (cBm IgM), y otra población que correspondería a células B de la zona marginal (cBZM) que circulan en sangre (Berkowska, et al., 2011a; Della Valle et al., 2014; Martin, et al., 2015; Reynaud, et al., 2012). Es importante diferenciar estas poblaciones tan disímiles no solo desde el punto de vista fundamental, para entender la ontogenia y la función de las células $\mathrm{B}$, sino porque en infecciones como la de rotavirus se podrían correlacionar diferencialmente con la protección después de la infección natural o la vacunación (Franco \& Greenberg, 2013).

\section{Las cBm IgM humanas y las controversias que suscitan}

El llegar a la conclusión de que las cBm IgM son una población heterogénea ha sido un proceso largo y complejo que se inició con su clasificación como cBm. Para la definición de cBm se han usado varias estrategias: inicialmente se llamó así a las células IgD- de larga vida, lo cual implicaba que habían sufrido conmutación de isotipo. Posteriormente, se observó que la expresión de CD27 en la superficie se correlacionaba con la capacidad de las células B de secretar inmunoglobulinas después de la estimulación policlonal (Agematsu, et al., 1997; Maurer, et al., 1992). Debido a la detección de células $\mathrm{CD}^{2} 7^{+} \mathrm{IgM}^{+}$capaces de secretar IgM bajo dichas condiciones, estos autores introdujeron, por primera vez, la noción de $\mathrm{cBm}$ IgM, la cual fue oficialmente propuesta con la identificación de hipermutaciones somáticas en los análisis moleculares de las porciones génicas que codifican para el $\operatorname{IgV}$ de células $\mathrm{CD}^{2} 7^{+} \mathrm{IgM}^{+}$(Klein, et al., 1997; Klein, et al., 1998; Tangye, et al., 1998). Además de la presencia de hipermutaciones somáticas, otros argumentos apoyan la noción de que las cBm IgM pertenecen a las subpoblaciones de memoria: 1) son raras en los niños, pero se acumulan con la edad (Agematsu, et al., 1997); 2) tienen larga vida (Richards, et al., 2000); 3) al igual que las $\mathrm{cBm}$ conmutadas, se diferencian como células secretoras de anticuerpos ante ciertos estímulos policlonales (Agematsu, et al., 1997; Himmelmann, et al., 2001; Shi, et al., 2003); 4) responden in vitro a estímulos normalmente derivados de linfocitos $\mathrm{T}$ de forma parecida a las $\mathrm{cBm}$ conmutadas y distinta a las células B vírgenes (Tangye, et al., 2003); 5) presentan mutaciones en el gen de la proteína 6 del linfoma de células B (Bcl6), lo que se considera una marca genética del paso por el centro germinal (Della Valle, et al., 2014; Seifert \& Kuppers, 2009), y 6) los análisis de los transcriptomas de $\mathrm{cBm}$ IgM, cBm con expresión solo de IgM y cBm conmutadas muestran que todas comparten un patrón de expresión génica global que refleja su función de células de memoria (aumento de la capacidad de respuesta al estímulo, del metabolismo, la proliferación y la diferenciación en plasmablastos), y que es diferente del patrón de las células B vírgenes (Seifert, et al., 2015).

Paralelamente, varios hallazgos sugieren que algunas $\mathrm{cBm}$ IgM también se generan por fuera de los centros germinales; los principales son: 1) se pueden encontrar niveles bajos de $\mathrm{cBm}$ IgM en humanos con inmunodeficiencias de CD40 o CD40L, en la cuales se considera que no hay formación de centros germinales (Agematsu, et al., 1998; Berkowska, et al., 2011a; Weller, et al., 2004; Weller, et al., 2001); 2) la AID también se ha identificado en células B extrafoliculares, lo que sugiere que el paso por el centro germinal no es una condición absoluta para la generación de hipermutaciones somáticas (Cattoretti, et al., 2006). Teniendo en cuenta algunas de estas evidencias, otros autores propusieron que las cBm IgM humanas son cBZM circulantes que poseen un repertorio de inmunoglobulinas diversificado por hipermutación generado en ausencia de una respuesta inmunitaria ante un antígeno, como ocurre en pollos, ovejas y conejos (Weller, et al., 2004; Weller, et al., 2008). Este planteamiento se ha visto sustentado por el hallazgo de la diversificación por hipermutación de los genes de inmunoglobulina en cBZM del bazo de niños antes del desarrollo de respuestas independientes de $\mathrm{T}$ funcionales (Bagnara, et al., 2015).

Durante varios años los hallazgos descritos parecieron contradictorios, pero recientemente diversos autores han propuesto que la población de $\mathrm{cBm}$ IgM circulante en humanos es una población heterogénea (Della Valle, et al., 2014; Martin, et al., 2015; Reynaud, et al., 2012). Existen por lo menos dos subpoblaciones: una de cBZM circulantes (Weller, et al., 2004), que como se dijo anteriormente tiene funciones similares a las innatas, y otra de cBm IgM con función adaptativa; ambas subpoblaciones pueden participar en respuestas inmunes dependientes e independientes de $\mathrm{T}$ (Cerutti, et al., 2013). Este aporte reciente dificulta mucho la interpretación de la mayoría de los estudios publicados hasta ahora, en los cuales se asumía que las cBm IgM humanas constituían una población homogénea.

\section{Las funciones de las cBm IgM}

Los estudios más detallados en ratones indican que las $\mathrm{cBm}$ IgM poseen un componente independiente de $\mathrm{T}$ y uno dependiente y que podrían ser muy relevantes en el funcionamiento de las células B de memoria (Dogan, et al., 2009; Kaji, et al., 2012; Link, et al., 2012; Pape, et al., 2011; Taylor, et al., 2012), porque se generan durante la respuesta inmunitaria primaria y durante la respuesta secundaria pueden reiniciar una reacción de centro germinal, dando origen a nuevas células IgG y posibilitando, así, el reaprovisionamiento del compartimiento de $\mathrm{cBm} \operatorname{IgM}$ y de $\mathrm{cBm} \operatorname{IgG}$ a partir de precursores que hayan tenido contacto previo con el antígeno (Dogan, et al., 2009). De forma similar, en un modelo de transferencia celular en ratones se mostró que las $\mathrm{cBm}$ IgM se producen después de la inmunización con un antígeno dependiente de $\mathrm{T}$ y cuando los títulos en suero de IgG específica de dicho antígeno han decaído las cBm IgM regresan al centro germinal para generar nuevas células secretoras de anticuerpos $\operatorname{IgG}$ (Pape, et al., 2011). Estos estudios señalan la existencia 
de múltiples "rondas" de respuestas de $\mathrm{cBm}$ en los ratones y un probable rol de la cBm IgM como reservorio para la generación de $\mathrm{cBm}$ conmutadas.

La funcionalidad de las células B se demostró en un modelo murino de infección bacteriana que indujo una numerosa población de $\mathrm{cBm}$ IgM localizada en la zona marginal del bazo y con características de células $\mathrm{B}$ de la respuesta adaptativa. Al eliminar in vivo estas $\mathrm{cBm}$ IgM disminuyeron las respuestas de memoria $\operatorname{IgG}$ ante un reto, lo cual demuestra que dicha población es importante en la memoria humoral y que las cBm IgM pueden cambiar de isotipo ante el reencuentro con el antígeno (Yates, et al., 2013).

Debido a la dificultad para estudiar ciertas repuestas inmunitarias en humanos, se ha recurrido a los modelos de ratones "humanizados", en los cuales se trasplantan células inmunitarias humanas a ratones inmunodeficientes SCID/ SCID y se los inmuniza con diferentes antígenos. Con uno de dichos modelos, se observó que cuando los ratones recibieron el trasplante de $\mathrm{cBm} \operatorname{IgM}$ y fueron inmunizados con Streptococcus pneumoniae inactivado o con vacuna contra el neumococo, hubo incrementos en la $\operatorname{IgM}$ y en la IgG específicas de antígenos independientes de $\mathrm{T}$, como el lipopolisacárido, o dependientes de $\mathrm{T}$, como las proteínas de la bacteria (Moens, et al., 2008). Con este mismo modelo, se demostró que las cBm IgM humanas trasplantadas conmutaron a IgG y disminuyeron la antigenemia y viremia por rotavirus en los ratones (Narváez, et al., 2012).

En humanos las cBm IgM tienen un papel importante en la protección contra antígenos independientes de $\mathrm{T}$ de bacterias encapsuladas como S. pneumoniae (Capolunghi, et al., 2008; Khaskhely, et al., 2012; Kruetzmann, et al., 2003). Se ha reportado una correlación entre la ausencia de cBm IgM y la falta de respuesta inmunitaria a bacterias encapsuladas, no solo en pacientes con extirpación del bazo sino también con bazos intactos (Rosado, et al., 2013). También se ha encontrado que la frecuencia de las cBm $\operatorname{IgM}$ disminuye con la edad y esto iría de la mano con el incremento en el riesgo de morbilidad y mortalidad debidas a neumonía por neumococo en los ancianos (Shi, et al., 2005). No obstante, en otro estudio no se observó dicha disminución (ColonnaRomano, et al., 2009).

Recientemente se reportaron $\mathrm{cBm} \operatorname{IgM}$ humanas específicas de toxoide tetánico y del antígeno eritrocítico $\mathrm{RhD}$ con signos de paso por el centro germinal (genes Bcl-6 mutados) (Della Valle, et al., 2014), lo que apoya la noción de que existen "rondas" de cBm en humanos y que las cBm IgM tienen larga vida y pueden reconformar el grupo de $\mathrm{cBm}$ conmutadas después de exposiciones repetidas al antígeno, como se ha descrito en ratones.

Hasta donde se sabe, existe poca evidencia directa del incremento de las cBm IgM en el curso de una infección humana (Kendall, et al., 2010). Estos autores estudiaron adultos con infección por Vibrio cholerae $\mathrm{O} 1$ y estimularon de forma policlonal células mononucleares de sangre periférica para inducir la transformación de las diferentes subpoblaciones de cBm en células secretoras de anticuerpos. En los sobrenadantes de cultivo encontraron un aumento significativo de las $\operatorname{IgM}$ específicas de un antígeno independiente de $\mathrm{T}$, un lipopolisacárido, y un antígeno dependiente de $\mathrm{T}$, la subunidad $\mathrm{B}$ de la toxina colérica, después de 30 días de infección (Kendall, et al., 2010). Sin embargo, bajo estas condiciones experimentales la IgM detectada puede provenir de $\mathrm{cBm}$ IgM o de $\mathrm{cBm}$ solo IgM.

En nuestro grupo de investigación se han venido estudiando las células B específicas de rotavirus (células B-RV) desde hace varios años con el ánimo de entender la respuesta de células B-RV y de proponer nuevos marcadores de protección después de la vacunación. Se han detectado frecuencias bajas de cBm IgM específicas de RV circulantes en lactantes que carecían de RV-IgA y que, por lo tanto, era improbable que hubieran sido infectados por el virus (Rojas, et al., 2007). Esto sugiere que se desarrollaron en ausencia de estimulación antigénica y que, posiblemente, hacían parte de las cBZM circulantes. En adultos sanos, probablemente infectados por RV en más de una ocasión, se evidenció que las células B-RV circulantes estaban enriquecidas en las cBm IgM (Herrera, et al., 2014; Rojas, et al., 2008). La antigenemia y la viremia podrían explicar el que las células $\mathrm{B}-\mathrm{RV}$ estuvieran enriquecidas en $\mathrm{cBm}$ IgM originadas en el bazo, pero queda por establecer cuál sería el papel de las células semejantes a las cBZM identificadas en el intestino de humanos (Spencer, et al., 1985).

Al analizar las células IgM que unen el antígeno de RV se observó que las cBm IgM se podían subdividir con base en la expresión relativa de cada una de las inmunoglobulinas en dos poblaciones: las que expresaban altos niveles de $\operatorname{IgM}$ y bajos niveles de $\operatorname{IgD}\left(\operatorname{IgM}^{++} \operatorname{IgD}{ }^{+}\right)$y las que expresaban bajos niveles de $\operatorname{IgM}$ y altos niveles de $\operatorname{IgD}$ $\left(\operatorname{IgM}^{+} \operatorname{IgD}^{++}\right)$(Narváez, et al., 2012). Recientemente se demostró que, comparadas con las $\mathrm{cBm}$ totales, las células $\mathrm{B}-\mathrm{RV}$ estaban enriquecidas en todas las poblaciones de cBm IgM: solo IgM, $\operatorname{IgM}^{++} \operatorname{IgD}^{+}$e $\operatorname{IgM}^{+} \operatorname{IgD}^{++}$(Herrera, et al., 2014). Además, se observó que al estimular in vitro la población de cBm IgM, aquellas células específicas de $\mathrm{RV}$ hicieron conmutación de isotipo en menor grado que la población total homóloga (Narváez, et al., 2012). Por último, las inmunoglobulinas con componente secretor específicas de RV presentes en plasma de niños vacunados con RIX 4414, un precursor de la vacuna Rotarix $\AA$, se correlacionaron con la protección (Herrera, et al., 2013). Parte de estas inmunoglobulinas con componente secretor son probablemente IgM (Herrera, et al., 2013), lo que sugiere que las cBm IgM cumplirían un papel importante en la protección frente a RV. Faltaría por establecer si alguna de estas subpoblaciones es más relevante para la protección. 


\section{Características de las células B de la zona marginal}

Las células B de la zona marginal (cBZM) del bazo de roedores se consideran un linaje separado que se diferencia de las células B transicionales T2 en precursores de zona marginal, entre otras razones por la influencia de NOTCH2, y que continúan la diferenciación hacia cBZM (Pillai \& Cariappa, 2009). La gran mayoría son vírgenes, tienen muy pocas mutaciones en sus genes de inmunoglobulinas (Hendricks, et al., 2011), están en un estado de pre-activación (Martin \& Kearney, 2002), desarrollan una respuesta rápida de $\operatorname{IgM}$ e $\operatorname{IgG} 3$ contra patógenos independientes de $\mathrm{T}$ que ingresan al compartimento sanguíneo (Reynaud, et al., 2012), y se las cataloga como células B de la respuesta inmunitaria innata. No obstante, son funcionalmente versátiles, pues responden a múltiples retos microbianos que incluyen también antígenos dependientes de T como las proteínas (Cerutti, et al., 2013). En contraste, además de las que se encuentran en el bazo de los humanos, existen equivalentes de cBZM en el área subepitelial de las placas de Peyer (Spencer, et al., 1985), en la pared interior de los senos subcapsulares de los nódulos linfáticos y en el epitelio de las criptas de las amígdalas (Cerutti, et al., 2013), así como circulantes en sangre (Weller, et al., 2004).

Las cBZM de bazos humanos comparten ciertas características con las cBZM de ratones: la localización en la periferia de los folículos B y el fenotipo: $\operatorname{IgM}^{++} \operatorname{IgD}^{+}$ $\mathrm{CD}^{++}{ }^{+C^{-}}$23- CD1c $^{++}$(Bagnara, et al., 2015; Ettinger, et al., 2007; Weller, et al., 2004), pero tienen un receptor de inmunoglobulinas mutado (Dunn-Walters, et al., 1995; Reynaud, et al., 2012) y diversificado por hipermutación durante la ontogenia (Weller, et al., 2004; Weller, et al., 2008). En efecto, se han detectado células con fenotipo de $\mathrm{cBm} \operatorname{IgM}$ con hipermutaciones somáticas durante el desarrollo fetal humano (Scheeren, et al., 2008). En cuanto al origen de la diversificación por hipermutación de los genes de las inmunoglobulinas de cBZM, hay datos contradictorios sobre el papel de los neutrófilos en su inducción (Nagelkerke, et al., 2014; Puga, et al., 2012). En dos estudios, uno en ratones (Wesemann, et al., 2013) y otro en humanos (Vossenkamper, et al., 2013), se ha sugerido que las células B pueden desarrollarse en la lámina propia del intestino, bajo la influencia de los microorganismos intestinales, lo que indica que este podría ser un sitio de diversificación del repertorio de las cBZM.

Recientemente se reportó la detección de precursores de cBZM en los bazos de niños (Descatoire, et al., 2014). Estos precursores se diferencian en células semejantes a cBZM ante el estímulo por la vía NOTCH2 y su ligando, DLL1. Además, el transcriptoma de cBZM difiere del de las células $B$ vírgenes del bazo en la expresión génica de por lo menos 1.855 genes, mientras que los precursores parecen compartir la expresión común a las dos poblaciones.
En un estudio reciente se analizaron muestras pareadas de sangre y bazo de tres individuos y se compararon las secuencias $\mathrm{V}_{\mathrm{H}} \mathrm{DJ}_{\mathrm{H}}$ de las cBZM del bazo y las cBm IgM circulantes (Bagnara, et al., 2015). Los autores evidenciaron, como era de esperarse, un alto porcentaje de clones compartidos entre estas dos poblaciones. Más aún, encontraron que en la circulación se localiza solamente un subgrupo relacionado con las cBZM de bazo y que, por lo tanto, no todas las cBZM de bazo vuelven a circular de igual forma (Bagnara, et al., 2015). Este hallazgo deja abierta la pregunta sobre el tipo de selección que se ejerce sobre las cBZM del bazo para que vuelvan a circular.

Cuando se contrastaron los transcriptomas de las $\mathrm{cBm} \mathrm{IgM}^{+}$ humanas de sangre y cBZM del bazo se encontró que se asemejaban, pero que eran muy diferentes al de las cBm conmutadas (Descatoire, et al., 2014). De los 50 genes con expresión más diferenciada entre las cBZM y las cBm conmutadas de bazo, solamente 35 también lo eran con respecto a las $\mathrm{cBm}$ IgM de sangre y las $\mathrm{cBm}$ conmutadas en sangre. Estos resultados indican que, aunque hay una gran homología entre las cBZM de bazo y las cBm IgMde sangre, existen diferencias entre ellas que podrían explicarse por la heterogeneidad de la población sanguínea y se deberían, en parte, a la presencia de $\mathrm{cBm}$ IgM de la respuesta adaptativa.

Comparadas con sus contrapartes conmutadas, los transcriptomas de las cBZM de bazo y las cBm IgM de sangre tienen una regulación positiva marcada del factor de transcripción SOX7 y una regulación negativa del gen $\mathrm{COCH}$ y de los factores de transcripción TOX y HOPX (Descatoire, et al., 2014). Todas estas moléculas tienen funciones centrales en el sistema inmunitario; SOX7, particularmente, es un factor de transcripción con un papel importante en el desarrollo de los precursores de células sanguíneas (Seguin, et al., 2008) $\mathrm{y}$ es el factor de transcripción que mejor discrimina las $\mathrm{cBm}$ conmutadas de las cBZM, por lo que se ha propuesto como el factor de transcripción clave en su desarrollo (Descatoire, et al., 2014).

Por último, es importante resaltar que pacientes con el síndrome de Alagille, que tienen una deficiencia de $\mathrm{NOTCH} 2$, presentan una reducción en el número de $\mathrm{cBm}$ IgM, pero mantienen niveles de $\mathrm{cBm}$ conmutadas normales. Sin embargo, en la sangre de los pacientes permanece aproximadamente una tercera parte de las $\mathrm{cBm}$ IgM presentes en individuos sanos, lo que respalda la noción de la heterogeneidad de esta población (Descatoire, et al., 2014).

\section{Características de la subpoblación de cBm IgM dependiente de centro germinal}

La existencia de un componente de $\mathrm{cBm}$ IgM dependiente de centro germinal en el seno de la población de cBm IgM humanas está respaldada por varios hallazgos. En dos estudios (Seifert \& Kuppers, 2009; Wu, et al., 2010) se compararon los patrones de mutaciones de los genes de las 
inmunoglobulinas de las $\mathrm{cBm} \operatorname{IgM}$ (sin incluir las cBm solo IgM) y de las $\mathrm{cBm}$ conmutadas circulantes en sangre: en un caso los resultados de la amplificación específica de CDR3 indicaron que ambas poblaciones se generaban en el centro germinal y se encontraron evidencias de que el desarrollo puede ser secuencial y que inicialmente se generan $\mathrm{cBm}$ $\operatorname{IgM}$, las cuales pueden salir tempranamente del centro germinal o permanecer en él y generar $\mathrm{cBm}$ conmutadas; por lo tanto, la población de $\mathrm{cBm}$ IgM y la de $\mathrm{cBm}$ conmutadas estarían relacionadas (Seifert \& Kuppers, 2009). En el otro caso, por el contrario, los patrones de mutaciones de los genes de las inmunoglobulinas de ambas poblaciones fueron muy distintos, lo que sugiere que en la mayoría de los casos las cBm IgM se desarrollan en respuesta a estímulos distintos a los que generan las $\mathrm{cBm}$ conmutadas (Wu, et al., 2010), aunque hay que tener en cuenta que esta última aproximación metodológica es menos informativa que la amplificación de los CDR3. En el primero de estos trabajos también se identificaron mutaciones en el gen Bcl6 en las $\mathrm{cBm}$ IgM humanas y, por lo tanto, se vio respaldada la noción de que algunas de estas son emigrantes tempranas del centro germinal de una respuesta clásica dependiente de linfocitos T (Seifert \& Kuppers, 2009).

Los análisis de la capacidad de migración in vitro muestran que, comparadas con las cBm conmutadas, las cBm IgM tienen mayor capacidad de migración al folículo B por presentar mayor sensibilidad a la quimiotaxis dependiente de CXCR5 y CCR7, expresar moléculas de adhesión celular C (JAM-C) y tener mayor capacidad de diferenciarse en el centro germinal debido a su incrementada inducción de expresión del Bcl-6 in vitro (Seifert, et al., 2015). Esto sugiere que tienen mayor propensión a volver a entrar en reacciones de centro germinal, y sustenta la existencia de "rondas" de respuestas de cBm humanas similares a las del ratón.

Al estudiar la historia de la replicación de las $\mathrm{cBm} \operatorname{IgM}$ (Berkowska, et al., 2011b), se ha detectado que las cBm solo IgM parecen haberse dividido en forma similar a las $\mathrm{cBm}$ conmutadas, pero más que las $\mathrm{cBm}$ IgM, por lo cual se espera que estas últimas tengan una mayor capacidad de replicación que las $\mathrm{cBm}$ solo IgM. Así mismo, el cocultivo con neutrófilos activados o estímulos como la terbutalina, la molécula de adhesión celular relacionada con el antígeno carcinoembrionario 8 (CEACAM8) o anti-Ig (estímulos independientes de linfocitos $\mathrm{T}$ ), inducen un mayor número de células que secretan anticuerpos en $\mathrm{cBm}$ IgM que en cBm conmutadas, en oposición a lo encontrado con los estímulos como el CD40 (estímulo dependiente de linfocitos T) (Seifert, et al., 2015). Últimamente se ha observado que las $\mathrm{cBm}$ IgM humanas purificadas circulantes en adultos sanos (incluidas las $\mathrm{cBm}$ solo IgM), y estimuladas de forma policlonal semejando una respuesta independiente de $\mathrm{T}$, proliferan rápidamente y generan células secretoras de anticuerpos IgG (Vásquez, et al., 2015).
Al comparar los transcriptomas de las $\mathrm{cBm} \operatorname{IgM}$ y las cBm conmutadas se identificaron 422 genes con expresión diferencial entre ellas y las proteínas codificadas en dichos genes explican algunos comportamientos biológicos de las cBm IgM in vitro (Seifert, et al., 2015).

Un factor de confusión muy importante al analizar los resultados de los diversos estudios sobre las cBm IgM, es que en varios de ellos las $\mathrm{cBm}$ solo $\operatorname{IgM}\left(\operatorname{IgD}^{-} \operatorname{IgM}^{+}\right)$están contenidas en la población de las cBm $\operatorname{IgM}\left(\operatorname{IgD}^{+} \operatorname{IgM}^{+}\right)$. Las $\mathrm{cBm}$ solo IgM, junto con las $\mathrm{cBm}$ conmutadas, se encuentran ausentes en pacientes con síndrome de hiper IgM ligada al cromosoma $\mathrm{X}$, lo que indica que se desarrollan por vías de diferenciación distintas de las $\mathrm{cBm} \operatorname{IgM}$ (Weller, et al., 2001). Esta es una de las razones por las cuales las cBm solo IgM se han considerado como las "verdaderas" cBm con fenotipo de IgM (Lanzavecchia \& Sallusto, 2009), y parecen generarse en centros germinales primarios (Berkowska, et al., 2011a).

Varios autores han comparado las cBm IgM con las cBm solo IgM. Al contrastar los patrones de longitud de los CDR3 de las células $B$ de sangre periférica humana se evidenció que mientras las células $\mathrm{B}$ vírgenes mostraban una gran diversidad en este parámetro, las $\mathrm{cBm}$ solo IgM y las cBm conmutadas presentaban una diversidad limitada que variaba según los individuos (Weill, et al., 2009). Llamó la atención que las $\mathrm{cBm}$ IgM exhibieron un patrón intermedio entre los dos patrones de longitud. Más aún, las cBm IgM tienen un repertorio de genes IGHV (Martin, et al., 2015) y de secuencias $\mathrm{V}_{\mathrm{H}} \mathrm{DJ}_{\mathrm{H}}$ (Bagnara, et al., 2015) diferente a las $\mathrm{cBm}$ solo IgM. Asimismo, al analizar las secuencias IGHJ se observó que las cBm IgM estaban más relacionadas con las $\mathrm{cBm}$ IgM CD27 que con las cBm solo IgM (Martin, et al., 2015). Además, las cBm solo IgM tienen mayor relación precursor-producto que las $\mathrm{cBm}$ conmutadas, lo que indica que representan células $\mathrm{B}$ derivadas del centro germinal (Bagnara, et al., 2015). En contraste, el análisis de los transcriptomas de las cBm IgM y las solo IgM evidencia que el único gen expresado de forma estadísticamente diferente es el IgD, lo que sugiere que tienen capacidades funcionales similares (Seifert, et al., 2015).

\section{Propuesta de procedimiento de diferenciación fenotípica de las subpoblaciones de cBm IgM}

Como ya se ha mencionado, en la mayoría de los estudios funcionales en humanos no es posible establecer cuál es el rol de cada una de las dos subpoblaciones de cBm IgM, para lo cual es necesario establecer su fenotipo. Muy recientemente, la subdivisión de las $\mathrm{cBm} \operatorname{IgM}$ que unen el antígeno de $\mathrm{RV}$ en $\operatorname{IgM}^{++} \operatorname{IgD}^{+}$e $\operatorname{IgM}^{+} \operatorname{IgD}^{++}$propuesta por Narváez, et al., 2012 fue retomada por un grupo inglés que analizó la expresión de diferentes marcadores en ambas poblaciones usando "citometría de masas" (Martin, et al., 2015). Esta técnica combina la citometría de flujo con la espectrometría de masas y permite el análisis de un mayor 
Tabla 1. Comparación de los marcadores expresados por las cBZM de bazo humanas y las dos subpoblaciones de cBm IgM circulantes en sangre periférica propuestas

\begin{tabular}{|c|c|c|c|c|c|}
\hline Marcador & cBZM & $\operatorname{IgM}^{++} \operatorname{IgD}^{+a}$ & $\operatorname{IgM}^{++} \operatorname{IgD}^{+b}$ & $\operatorname{IgM}^{+} \operatorname{IgD}^{++b}$ & Nombre/grupo \\
\hline CD35 & & & ++ & + & Receptor del complemento tipo 1, CR1 \\
\hline CD44 & & ++ & ++ & + & Antígeno Hermes, Pgp-1 \\
\hline $\mathrm{CD} 74$ & & & ++ & + & Cadena invariante del CMH de clase II \\
\hline $\mathrm{CD} 24$ & & & + & - & Antígeno estable al calor, glicoproteína \\
\hline $\mathrm{CD} 40$ & & & - & + & Receptor de coestimulación de cB \\
\hline CD23 & & - & - & + & FceRII \\
\hline Beta7 & & & $-/+$ & + & Integrina \\
\hline $\mathrm{CD} 21$ & & ++ & + & ++ & Receptor para C3d y EBV, forma el correceptor B con CD19 y CD81 \\
\hline CD38 & & & - & + & NAD glicohidrolasa \\
\hline CD95 & & + & + & - & Apo-1, Fas \\
\hline CXCR4 & & - & - & $-/+$ & Receptor para el factor derivado del estroma 1 (SDF-1) \\
\hline $\mathrm{TNF} \alpha$ & & & + & - & Citocina \\
\hline CD69 & & - & - & + & Molécula inductora de activación (AIM) \\
\hline CD27 & & ++ & ++ & + & Coestimulador de $\mathrm{cB}$ \\
\hline
\end{tabular}

a (Ettinger, et al., 2007); ${ }^{b}$ (Martin, et al., 2015)

número de parámetros celulares que la citometría de flujo convencional. Los autores encontraron que la frecuencia relativa de las células $\mathrm{B} \operatorname{IgM}^{+} \operatorname{IgD}^{++}$disminuía con la edad $\mathrm{y}$, por el contrario, la frecuencia relativa de las células B $\mathrm{IgM}^{++} \operatorname{IgD}^{+}$aumentaba con la edad (Martin, et al., 2015). Detectaron, además, algunos marcadores que se expresaban diferencialmente en estas dos subpoblaciones (Tabla1) y notaron que las células $\mathrm{B} \operatorname{IgM}^{++} \operatorname{IgD}^{+}$tuvieron más marcadores en común con las $\mathrm{cBm}$ solo $\mathrm{IgM}$ y con las $\mathrm{cBm}$ conmutadas que con las células B $\operatorname{IgM}^{+} \operatorname{IgD}^{++}$(Martin, et al., 2015). Por esta razón, los autores propusieron que las $\mathrm{cBm}$ IgM se pueden dividir en dos subpoblaciones: las células B $\operatorname{IgM}^{++} \operatorname{IgD}^{+}$podrían ser la población más semejante a las cBm solo IgM, dependientes de $\mathrm{T}$ (de centro germinal), $\mathrm{y}$ las células $\mathrm{B} \operatorname{IgM}^{+} \operatorname{IgD}^{++}$, la población responsable de la respuesta independiente de $\mathrm{T}$ contra los polisacáridos $\mathrm{y}$, por consiguiente, correspondiente a cBZM (Martin, et al., 2015). No obstante, esta interpretación es contraria al hecho de que las cBZM de bazo se han catalogado persistentemente como $\mathrm{IgM}^{++} \mathrm{IgD}^{+}$y comparten algunos marcadores con las células B $\operatorname{IgM}^{++} \operatorname{IgD}^{+}$(Tabla 1). Solo con más investigaciones, por ejemplo sobre el repertorio de genes de inmunoglobulinas, el transcriptoma y los estudios funcionales (proliferación y generación de células secretoras de anticuerpos), se podrá generar evidencia que respalde esta interpretación.

\section{Conclusión}

En la literatura científica reciente se comenzó a aceptar que los resultados aparentemente contradictorios del estudio de las cBm IgM circulantes en sangre se deben probablemente a que es una población heterogénea e importante para las respuestas de defensa de primera línea y para las de centro germinal. Aunque se ha propuesto que la expresión diferencial de $\operatorname{IgM}$ e $\operatorname{IgD}$ en las cBm $\operatorname{IgM}$ serviría para distinguir estas subpoblaciones de células $\mathrm{B}$, se requieren más estudios fenotípicos y funcionales para esclarecer sus características.

\section{Agradecimientos}

A los doctores Manuel A. Franco y Daniel Herrera, por la lectura crítica de este manuscrito; a Camilo Vásquez, por la elaboración de la figura, y a la Pontificia Universidad Javeriana por el apoyo durante la escritura del manuscrito.

\section{Conflicto de intereses}

Declaro no tener conflicto de intereses.

\section{Bibliografía}

Agematsu, K., Nagumo, H., Shinozaki, K., Hokibara, S., Yasui, K., Terada, K., Kawamura, N., Toba, T., Nonoyama, S., Ochs, H.D., Komiyama, A. (1998). Absence of IgD$\mathrm{CD} 27(+)$ memory B cell population in X-linked hyper-IgM syndrome. J Clin Invest 102 (4): 853-860.

Agematsu, K., Nagumo, H., Yang, F.C., Nakazawa, T., Fukushima, K., Ito, S., Sugita, K., Mori, T., Kobata, T., Morimoto, C., Komiyama,A. (1997). B cell subpopulations separated by CD27 and crucial collaboration of CD27+ B cells and helper T cells in immunoglobulin production. Eur J Immunol. 27 (8): 2073-2079.

Bagnara, D., Squillario, M., Kipling, D., Mora, T., Walczak, A.M., Da Silva, L., Weller, S., Dunn-Walters, D.K., Weill, J.C., Reynaud, C.A. (2015). A reassessment of IgM memory subsets in humans. J Immunol. 195 (8): 3716-3724.

Baumgarth, N. (2011). The double life of a B-1 cell: Self-reactivity selects for protective effector functions. Nat Rev Immunol. 11 (1): 34-46. 
Berkowska, M.A., Driessen, G.J., Bikos, V., GrosserichterWagener, C., Stamatopoulos, K., Cerutti, A., He, B., Biermann, K., Lange, J.F., van der Burg, M., van Dongen, J.J., van Zelm, M.C. (2011a). Human memory B cells originate from three distinct germinal centerdependent and -independent maturation pathways. Blood. 118 (8): 2150-2158.

Berkowska, M.A., van der Burg, M., van Dongen, J.J., van Zelm, M.C. (2011b). Checkpoints of B cell differentiation: Visualizing Ig-centric processes. Ann N Y Acad Sci. 1246: 11-25.

Capolunghi, F., Cascioli, S., Giorda, E., Rosado, M.M., Plebani, A., Auriti, C., Seganti, G., Zuntini, R., Ferrari, S., Cagliuso, M., Quinti, I., Carsetti, R. (2008). CpG drives human transitional $\mathrm{B}$ cells to terminal differentiation and production of natural antibodies. J Immunol. 180 (2): 800-808.

Cattoretti, G., Buttner, M., Shaknovich, R., Kremmer, E., Alobeid, B., Niedobitek, G. (2006). Nuclear and cytoplasmic AID in extrafollicular and germinal center B cells. Blood. 107 (10): 3967-3975.

Cerutti, A., Cols, M., Puga, I. (2013). Marginal zone B cells: Virtues of innate-like antibody-producing lymphocytes. Nat Rev Immunol. 13 (2): 118-132.

Chen, K., Cerutti, A. (2010). New insights into the enigma of immunoglobulin D. Immunol Rev. 237 (1): 160-179.

Colonna-Romano, G., Bulati, M., Aquino, A., Pellicano, M., Vitello, S., Lio, D., Candore, G., Caruso, C. (2009). A double-negative (IgD-CD27-) B cell population is increased in the peripheral blood of elderly people. Mech Ageing Dev. 130 (10): 681-690.

Della Valle, L., Dohmen, S.E., Verhagen, O.J., Berkowska, M.A., Vidarsson, G., Ellen van der Schoot, C. (2014). The majority of human memory $\mathrm{B}$ cells recognizing $\mathrm{RhD}$ and tetanus resides in IgM+ B cells. J Immunol. 193 (3): 1071-1079.

Descatoire, M., Weller, S., Irtan, S., Feuillard, J., Storck, S., Guiochon-Mantel, A., Bouligand, J., Morali, A., Cohen, J., Jacquemin, E., Iascone, M., Bole-Feysot, C., Cagnard, N., Weill, J.C., Reynaud, C.A. (2014). Identification of a human splenic marginal zone B cell precursor with NOTCH2-dependent differentiation properties. J Exp Med. 211 (5): 987-1000.

Dogan, I., Bertocci, B., Vilmont, V., Delbos, F., Megret, J., Storck, S., Reynaud, C.A., Weill, J.C. (2009). Multiple layers of B cell memory with different effector functions. Nat Immunol. 10 (12): 1292-1299.

Dunn-Walters, D.K., Isaacson, P.G., Spencer, J. (1995). Analysis of mutations in immunoglobulin heavy chain variable region genes of microdissected marginal zone (MGZ) B cells suggests that the MGZ of human spleen is a reservoir of memory B cells. J Exp Med. 182 (2): 559-566.

Ettinger, R., Sims, G.P., Robbins, R., Withers, D., Fischer, R.T., Grammer, A.C., Kuchen, S., Lipsky, P.E. (2007). IL21 and BAFF/BLyS synergize in stimulating plasma cell differentiation from a unique population of human splenic memory B cells. J Immunol. 178 (5): 2872-2882.

Fecteau, J.F. \& Neron, S. (2003). CD40 stimulation of human peripheral B lymphocytes: Distinct response from naive and memory cells. J Immunol. 171 (9): 4621-4629.

Franco, M. \& Greenberg, H. (2013). Rotavirus. Microbiol Spectrum. 1 (2).

Hendricks, J., Visser, A., Dammers, P.M., Burgerhof, J.G., Bos, N.A., Kroese, F.G. (2011). Class-switched marginal zone $\mathrm{B}$ cells in spleen have relatively low numbers of somatic mutations. Mol Immunol. 48 (6-7): 874-882.

Herrera, D., Rojas, O.L., Duarte-Rey, C., Mantilla, R.D., Ángel, J., Franco, M.A. (2014). Simultaneous assessment of rotavirus-specific memory B cells and serological memory after B cell depletion therapy with rituximab. PLoS One. 9 (5): e97087.

Herrera, D., Vásquez, C., Corthésy, B., Franco, M.A., Ángel, J. (2013). Rotavirus specific plasma secretory immunoglobulin in children with acute gastroenteritis and children vaccinated with an attenuated human rotavirus vaccine. Hum Vaccin Immunother. 9 (11): 2409-2417.

Himmelmann, A., Gautschi, O., Nawrath, M., Bolliger, U., Fehr, J., Stahel, R.A. (2001). Persistent polyclonal B-cell lymphocytosis is an expansion of functional $\operatorname{IgD}(+)$ CD27(+) memory B cells. Br J Haematol. 114 (2): 400-405.

Jackson, S.M., Wilson, P.C., James, J.A., Capra, J.D. (2008). Human B cell subsets. Adv Immunol. 98: 151-224.

Kaji, T., Ishige, A., Hikida, M., Taka, J., Hijikata, A., Kubo, M., Nagashima, T., Takahashi, Y., Kurosaki, T., Okada, M., Ohara, O., Rajewsky, K., Takemori, T. (2012). Distinct cellular pathways select germline-encoded and somatically mutated antibodies into immunological memory. J Exp Med. 209 (11): 2079-2097.

Kendall, E.A., Tarique, A.A., Hossain, A., Alam, M.M., Arifuzzaman, M., Akhtar, N., Chowdhury, F., Khan, A.I., Larocque, R.C., Harris, J.B., Ryan, E.T., Qadri, F., Calderwood, S.B. (2010). Development of immunoglobulin $\mathrm{M}$ memory to both a T-cell-independent and a T-cell-dependent antigen following infection with Vibrio cholerae $\mathrm{O} 1$ in Bangladesh. Infect Immun. 78 (1): 253-259.

Khaskhely, N., Mosakowski, J., Thompson, R.S., Khuder, S., Smithson, S.L., Westerink, M.A. (2012). Phenotypic analysis of pneumococcal polysaccharide-specific B cells. J Immunol. 188 (5): 2455-2463.

Klein, U., Kuppers, R., Rajewsky, K. (1997). Evidence for a large compartment of IgM-expressing memory B cells in humans. Blood. 89 (4): 1288-1298.

Klein, U., Rajewsky, K., Kuppers, R. (1998). Human immunoglobulin $(\mathrm{Ig}) \mathrm{M}+\mathrm{IgD}+$ peripheral blood $\mathrm{B}$ cells expressing the CD27 cell surface antigen carry somatically mutated variable region genes: $\mathrm{CD} 27$ as a general marker for somatically mutated (memory) B cells. J Exp Med. 188 (9): 1679-1689. 
Kruetzmann, S., Rosado, M.M., Weber, H., Germing, U., Tournilhac, O., Peter, H.H., Berner, R., Peters, A., Boehm, T., Plebani, A., Quinti, I., Carsetti, R. (2003). Human immunoglobulin M memory B cells controlling Streptococcus pneumoniae infections are generated in the spleen. J Exp Med. 197 (7): 939-945.

Lanzavecchia, A. \& Sallusto, F. (2009). Human B cell memory. Curr Opin Immunol. 21 (3): 298-304.

Link, A., Zabel, F., Schnetzler, Y., Titz, A., Brombacher, F., Bachmann, M.F. (2012). Innate immunity mediates follicular transport of particulate but not soluble protein antigen. J Immunol. 188 (8): 3724-3733.

Martin, F. \& Kearney, J.F. (2002). Marginal-zone B cells. Nat Rev Immunol. 2 (5): 323-335.

Martin, V., Wu, Y.C., Kipling, D., Dunn-Walters, D.K. (2015). Age-related aspects of human IgM B cell heterogeneity. Ann N Y Acad Sci. 1361 (1): 153-163

Maurer, D., Fischer, G.F., Fae, I., Majdic, O., Stuhlmeier, K., Von Jeney, N., Holter, W., Knapp, W. (1992). IgM and IgG but not cytokine secretion is restricted to the CD27+ B lymphocyte subset. J Immunol. 148 (12): 3700-3705.

Moens, L., Wuyts, G., Boon, L., den Hartog, M.T., Ceuppens, J.L., Bossuyt, X. (2008). The human polysaccharideand protein-specific immune response to Streptococcus pneumoniae is dependent on $\mathrm{CD} 4(+) \mathrm{T}$ lymphocytes, $\mathrm{CD} 14(+)$ monocytes, and the CD40-CD40 ligand interaction. The Journal of allergy and clinical immunology. 122 (6): 1231-1233.

Mroczek, E.S., Ippolito, G.C., Rogosch, T., Hoi, K.H., Hwangpo, T.A., Brand, M.G., Zhuang, Y., Liu, C.R., Schneider, D.A., Zemlin, M., Brown, E.E., Georgiou, G., Schroeder, H.W., Jr. (2014). Differences in the composition of the human antibody repertoire by B cell subsets in the blood. Frontiers in immunology. 5: 96.

Nagelkerke, S.Q., aan de Kerk, D.J., Jansen, M.H., van den Berg, T.K., Kuijpers, T.W. (2014). Failure to detect functional neutrophil B helper cells in the human spleen. PLoS One. 9 (2): e88377.

Narváez, C.F., Feng, N., Vásquez, C., Sen, A., Ángel, J., Greenberg, H.B., Franco, M.A. (2012). Human rotavirusspecific IgM Memory B cells have differential cloning efficiencies and switch capacities and play a role in antiviral immunity in vivo. J Virol. 86 (19): 10829-10840.

Pape, K.A., Taylor, J.J., Maul, R.W., Gearhart, P.J., Jenkins, M.K. (2011). Different B cell populations mediate early and late memory during an endogenous immune response. Science. 331 (6021): 1203-1207.

Pascual, V., Liu, Y.J., Magalski, A., de Bouteiller, O., Banchereau, J., Capra, J.D. (1994). Analysis of somatic mutation in five B cell subsets of human tonsil. J Exp Med. 180 (1): 329-339.

Pillai, S. \& Cariappa, A. (2009). The follicular versus marginal zone B lymphocyte cell fate decision. Nat Rev Immunol. 9 (11): 767-777.
Puga, I., Cols, M., Barra, C.M., He, B., Cassis, L., Gentile, M., Comerma, L., Chorny, A., Shan, M., Xu, W., Magri, G., Knowles, D.M., Tam, W., Chiu, A., Bussel, J.B., Serrano, S., Lorente, J.A., Bellosillo, B., Lloreta, J., Juanpere, N., Alameda, F., Baro, T., de Heredia, C.D., Toran, N., Catala, A., Torrebadell, M., Fortuny, C., Cusi, V., Carreras, C., Díaz, G.A., Blander, J.M., Farber, C.M., Silvestri, G., Cunningham-Rundles, C., Calvillo, M., Dufour, C., Notarangelo, L.D., Lougaris, V., Plebani, A., Casanova, J.L., Ganal, S.C., Diefenbach, A., Arostegui, J.I., Juan, M., Yague, J., Mahlaoui, N., Donadieu, J., Chen, K., Cerutti, A. (2012). B cell-helper neutrophils stimulate the diversification and production of immunoglobulin in the marginal zone of the spleen. Nat Immunol. 13 (2): 170-180.

Reynaud, C.A., Descatoire, M., Dogan, I., Huetz, F., Weller, S., Weill, J.C. (2012). IgM memory B cells: a mouse/human paradox. Cell Mol Life Sci. 69 (10): 1625-1634.

Reynaud, C.A. \& Weill, J.C. (2012). Gene profiling of CD11b and CD11b B1 cell subsets reveals potential cell sorting artifacts. J Exp Med. 209 (3): 433-434; author reply: 434-436.

Richards, S.J., Morgan, G.J., Hillmen, P. (2000). Immunophenotypic analysis of B cells in $\mathrm{PNH}$ : Insights into the generation of circulating naive and memory B cells. Blood. 96 (10): 3522-3528.

Rojas, O.L., Caicedo, L., Guzmán, C., Rodríguez, L.S., Castañeda, J., Uribe, L., Andrade, Y., Pinzón, R., Narváez, C.F., Lozano, J.M., De Vos, B., Franco, M.A., Ángel, J. (2007). Evaluation of circulating intestinally committed memory B cells in children vaccinated with attenuated human rotavirus vaccine. Viral Immunol. 20 (2): 300-311.

Rojas, O.L., Narváez, C.F., Greenberg, H.B., Ángel, J., Franco, M.A. (2008). Characterization of rotavirus specific B cells and their relation with serological memory. Virology. $\mathbf{3 8 0}$ (2): 234-242.

Rosado, M.M., Gesualdo, F., Marcellini, V., Di Sabatino, A., Corazza, G.R., Smacchia, M.P., Nobili, B., Baronci, C., Russo, L., Rossi, F., Vito, R.D., Nicolosi, L., Inserra, A., Locatelli, F., Tozzi, A.E., Carsetti, R. (2013). Preserved antibody levels and loss of memory B cells against pneumococcus and tetanus after splenectomy: Tailoring better vaccination strategies. Eur J Immunol. 43 (10): 2659-2670.

Rothstein, T.L. \& Quach, T.D. (2015). The human counterpart of mouse B-1 cells. Ann N Y Acad Sci. 1362 (1): 143-162

Scheeren, F.A., Nagasawa, M., Weijer, K., Cupedo, T., Kirberg, J., Legrand, N., Spits, H. (2008). T cell-independent development and induction of somatic hypermutation in human IgM+ IgD+ CD27+ B cells. J Exp Med. 205 (9): 2033-2042.

Seguin, C.A., Draper, J.S., Nagy, A., Rossant, J. (2008). Establishment of endoderm progenitors by SOX transcription factor expression in human embryonic stem cells. Cell Stem Cell. 3 (2): 182-195.

Seifert, M. \& Kuppers, R., (2009). Molecular footprints of a germinal center derivation of human $\operatorname{IgM}+(\operatorname{IgD}+) \mathrm{CD} 27+$ $\mathrm{B}$ cells and the dynamics of memory B cell generation. J Exp Med. 206 (12): 2659-2669. 
Seifert, M., Przekopowitz, M., Taudien, S., Lollies, A., Ronge, V., Drees, B., Lindemann, M., Hillen, U., Engler, H., Singer, B.B., Kuppers, R. (2015). Functional capacities of human IgM memory B cells in early inflammatory responses and secondary germinal center reactions. Proc Natl Acad Sci USA. 112 (6): E546-555.

Shi, Y., Agematsu, K., Ochs, H.D., Sugane, K. (2003). Functional analysis of human memory B-cell subpopulations: $\mathrm{IgD}+\mathrm{CD} 27+\mathrm{B}$ cells are crucial in secondary immune response by producing high affinity IgM. Clin Immunol. 108 (2): 128-137.

Shi, Y., Yamazaki, T., Okubo, Y., Uehara, Y., Sugane, K., Agematsu, K. (2005). Regulation of aged humoral immune defense against pneumococcal bacteria by IgM memory B cell. J Immunol. 175 (5): 3262-3267.

Spencer, J., Finn, T., Pulford, K.A., Mason, D.Y., Isaacson, P.G. (1985). The human gut contains a novel population of B lymphocytes which resemble marginal zone cells. Clin Exp Immunol. 62 (3): 607-612.

Takemori, T., Kaji, T., Takahashi, Y., Shimoda, M., Rajewsky, K. (2014). Generation of memory B cells inside and outside germinal centers. Eur J Immunol. 44 (5): 1258-1264.

Tangye, S.G., Avery, D.T., Deenick, E.K., Hodgkin, P.D. (2003). Intrinsic differences in the proliferation of naive and memory human $\mathrm{B}$ cells as a mechanism for enhanced secondary immune responses. J Immunol. 170 (2): 686-694.

Tangye, S.G. \& Good, K.L. (2007). Human IgM+CD27+ B cells: memory B cells or "memory" B cells? J Immunol. 179 (1): 13-19.

Tangye, S.G., Liu, Y.J., Aversa, G., Phillips, J.H., de Vries, J.E. (1998). Identification of functional human splenic memory B cells by expression of CD148 and CD27. J Exp Med. 188 (9): 1691-1703.

Taylor, J.J., Pape, K.A., Jenkins, M.K. (2012). A germinal center-independent pathway generates unswitched memory B cells early in the primary response. J Exp Med. 209 (3): 597-606.

Vásquez, C., Franco, M.A., Ángel, J. (2015). Rapid proliferation and differentiation of a subset of circulating IgM memory $\mathrm{B}$ cells to a $\mathrm{CpG} /$ Cytokine stimulus in vitro. PLoS One. 10 (10): $\mathrm{e} 0139718$.
Vossenkamper, A., Blair, P.A., Safinia, N., Fraser, L.D., Das, L., Sanders, T.J., Stagg, A.J., Sanderson, J.D., Taylor, K., Chang, F., Choong, L.M., D’Cruz, D.P., Macdonald, T.T., Lombardi, G., Spencer, J. (2013). A role for gutassociated lymphoid tissue in shaping the human B cell repertoire. J Exp Med. 210 (9): 1665-1674.

Weill, J.C., Weller, S., Reynaud, C.A. (2009). Human marginal zone B cells. Annu Rev Immunol. 27: 267-285.

Weller, S., Braun, M.C., Tan, B.K., Rosenwald, A., Cordier, C., Conley, M.E., Plebani, A., Kumararatne, D.S., Bonnet, D., Tournilhac, O., Tchernia, G., Steiniger, B., Staudt, L.M., Casanova, J.L., Reynaud, C.A., Weill, J.C. (2004). Human blood IgM "memory" B cells are circulating splenic marginal zone B cells harboring a prediversified immunoglobulin repertoire. Blood. 104 (12): 3647-3654.

Weller, S., Faili, A., Garcia, C., Braun, M.C., Le Deist, F.F., de Saint Basile, G.G., Hermine, O., Fischer, A., Reynaud, C.A., Weill, J.C. (2001). CD40-CD40L independent Ig gene hypermutation suggests a second B cell diversification pathway in humans. Proc Natl Acad Sci USA. 98 (3): 1166-1170.

Weller, S., Mamani-Matsuda, M., Picard, C., Cordier, C., Lecoeuche, D., Gauthier, F., Weill, J.C., Reynaud, C.A. (2008). Somatic diversification in the absence of antigendriven responses is the hallmark of the $\mathrm{IgM}+\mathrm{IgD}+\mathrm{CD} 27+$ B cell repertoire in infants. J Exp Med. 205 (6): 1331-1342.

Wesemann, D.R., Portuguese, A.J., Meyers, R.M., Gallagher, M.P., Cluff-Jones, K., Magee, J.M., Panchakshari, R.A., Rodig, S.J., Kepler, T.B., Alt, F.W. (2013). Microbial colonization influences early B-lineage development in the gut lamina propria. Nature. 501 (7465): 112-115.

Wirths, S. \& Lanzavecchia, A. (2005). ABCB1 transporter discriminates human resting naive $\mathrm{B}$ cells from cycling transitional and memory B cells. Eur J Immunol. 35 (12): 3433-3441.

Wu, Y.C., Kipling, D., Leong, H.S., Martin, V., Ademokun, A.A., Dunn-Walters, D.K. (2010). High-throughput immunoglobulin repertoire analysis distinguishes between human IgM memory and switched memory B-cell populations. Blood. 116 (7): 1070-1078.

Yates, J.L., Racine, R., McBride, K.M., Winslow, G.M. (2013). $\mathrm{T}$ cell-dependent IgM memory B cells generated during bacterial infection are required for $\mathrm{IgG}$ responses to antigen challenge. J Immunol. 191 (3): 1240-1249. 